\title{
Ultra-Compact Neural Network ADC Exploiting Ferroelectric
} FET

This paper was downloaded from TechRxiv (https://www.techrxiv.org).

\section{LICENSE}

CC BY 4.0

SUBMISSION DATE / POSTED DATE

03-02-2022 / 11-02-2022

\section{CITATION}

Banerjee, Ayan; Bhattacharya, Sagnik; Chauhan, Yogesh Singh; Sahay, Shubham (2022): Ultra-Compact Neural Network ADC Exploiting Ferroelectric FET. TechRxiv. Preprint. https://doi.org/10.36227/techrxiv.19119269.v1

$\mathrm{DOI}$

10.36227/techrxiv.19119269.v1 


\title{
Ultra-Compact Neural Network ADC Exploiting Ferroelectric FETs
}

\author{
Ayan Banerjee*, Sagnik Bhattacharya*, Yogesh Singh Chauhan, Fellow, IEEE, and Shubham Sahay, Member, \\ IEEE
}

\begin{abstract}
Development of ultra-compact, low-to-medium precision analog-to-digital converters (ADCs) with unprecedented energy-efficiency is essential to meet the ever-increasing demand for data converters in advanced computing systems including neuromorphic accelerators based on emerging non-volatile memories. To this end, in this work, for the first time, we propose a feedforward neural network ADC based on a network of highly scalable, CMOS-compatible, and energy-efficient ferroelectricFinFET (Fe-FinFET) synaptic elements. Our lower triangular neural network (LTNN) ADC design, implemented using 7-nm technology from ARM along with an experimentally calibrated compact model for Fe-FinFETs, consumes $5.44 \mu \mathrm{W}$ of power, $1.03 \mu \mathrm{m}^{2}$ of area while operating at a speed of 1.23 megasamples per second for 4-bit precision. The proposed neural network ADC may pave the way for realization of highly efficient neuromorphic processing engines and neuro-optimizers based on cross-point array of emerging non-volatile memories.
\end{abstract}

Index Terms-Hopfield neural network, Ferroelectric FinFET, ADC, Feedforward, Successive approximation.

\section{INTRODUCTION}

A NALOG-to-digital converters (ADCs) form an integral part of the internet-of-things (IoT) ecosystem with a wide range of applications ranging from mobile IoT devices, wireless sensor nodes, communication systems including receivers and base stations, Ethernet, modems, DSL to video processing systems in HDTVs and CCD/ultrasonic medical imaging systems. Moreover, ADCs are inevitable while designing compute-in-memory primitives such as inference/multiplyaccumulate (MAC) accelerators based on the cross-point array of emerging non-volatile memories [1]-[5]. ADCs are required at each column of the cross-point array and often dominate the area and energy landscape of such neuromorphic processing engines limiting their area- and energy-efficiency, and the computational precision [6]-[9]. Therefore, there is an urgent need for ultra-compact and energy-efficient ADCs to realize the true potential of the advanced computing and communication systems.

A neural circuit consisting of a (synaptic) network of analog processors (neurons/decision elements), also known as the Hopfield neural network, with capability of solving optimization problems including decision/decomposition problems and linear programming problems was proposed in [10]. Furthermore, a simple ADC design utilizing the Hopfield neural

A. Banerjee, S. Bhattacharya, Y. S. Chauhan and S. Sahay are with the Department of Electrical Engineering, Indian Institute of Technology Kanpur, Kanpur 208016, India (email: ssahay@iitk.ac.in). This work was partially supported by the Semiconductor Research Corporation (SRP Task 3056.001) and Swarnajayanti fellowship (DST/SJF/ETA/02/17-18).

* A. Banerjee and S. Bhattacharya contributed equally to this work. network was also proposed [10]. However, the Hopfield ADC output exhibits hysteresis if the inputs are not reset before each calculation and tends to settle at local minima leading to spurious states and degradation in the conversion accuracy [10], [11]. Moreover, the variations in the input capacitance and the delay of neurons may lead to oscillatory behavior in Hopfield ADCs [12]. To mitigate the spurious outputs and oscillations arising due to the feedback in Hopfield neural networks, a feedforward lower triangular neural network (LTNN) ADC based on the successive approximation technique was proposed [11]. The LTNN ADC can be considered as the neural circuit equivalent of the SAR ADC.

However, the number of synaptic elements in a LTNN ADC increases quadratically with the ADC resolution $(n(n-1) / 2$ for $n$-bit LTNN ADC). Moreover, the synaptic elements must exhibit a high dynamic range $\left(O\left(2^{n}\right)\right)$ and support precise conductance-state tuning. While the digital implementation of such synaptic elements incurs a significant area and energy overhead, the analog CMOS implementations are susceptible to mismatch and process variations [13]. The emerging analog-grade non-volatile memories such as phase change memory (PCM), resistive RAMs (RRAMs), etc. appear promising for implementation of synaptic elements owing to their low footprint, CMOS-compatibility, and ability to tune the conductance-state using program-verify algorithm. Therefore, Hopfield neural networks utilizing PCM devices and RRAMs as synaptic elements were proposed recently [12], [14]-[18]. However, while the PCM devices exhibit a large cell current degrading the energy-efficiency of the synaptic network, RRAM devices suffer from a large temporal and spatial variability and their dynamic range is limited [8], [19]. Moreover, large scale back-end-of-line (BEOL) CMOSintegration of these NVMs introduces significant fabrication complexity and poses a technological challenge.

The recent discovery of ferroelectricity in doped-hafnium oxide stacks has propelled the development of ferroelectric FET based memories for storage. Considering the high scalability, ease of large scale CMOS integration, polarization tuning capability utilizing gate and drain program/erase schemes and ultra-low power consumption of the ferroelectric memories [20]-[23], it becomes imperative to explore their application as synaptic elements in neural circuits. Moreover, although several Hopfield ADC implementations utilizing digital, thin film, metal interconnects and PCM/RRAM based synaptic elements have been explored [12], [18], to the best of our knowledge, the hardware implementation of a feedforward LTNN ADC is still elusive.

To this end, in this work, for the first time, we pro- 


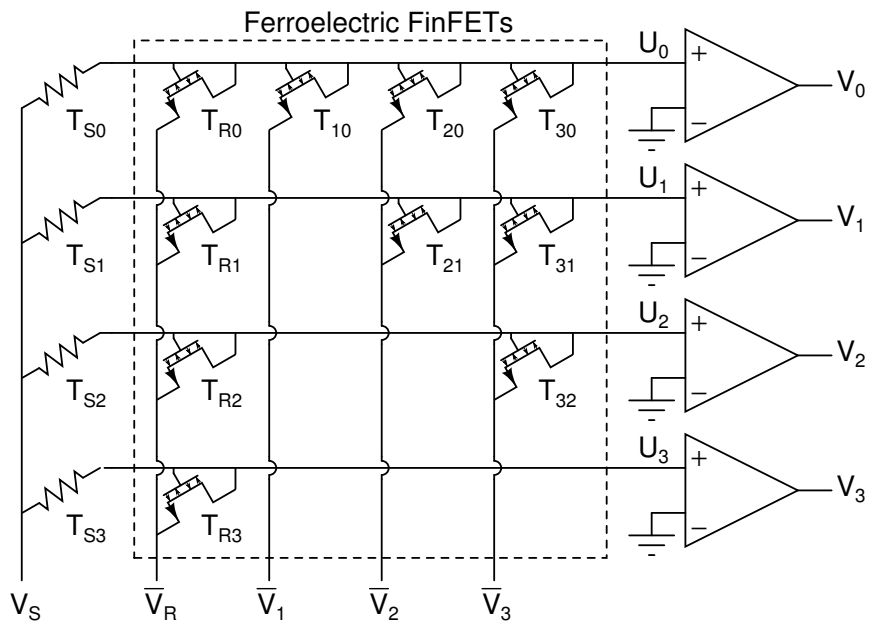

Fig. 1. Architecture of a 4-bit LTNN ADC using Fe-FinFET synapses.

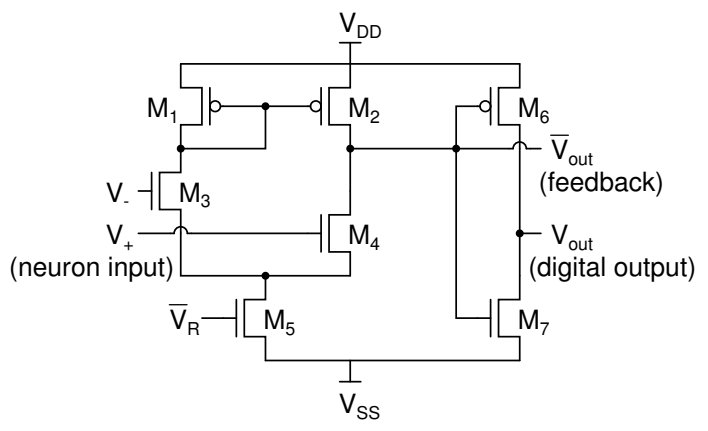

Fig. 2. The proposed neuron circuitry.

pose a feedforward neural network ADC with a network of ferroelectric-FinFET (Fe-FinFET) synapses based on dopedhafnium oxide stack. We also design a neuron circuit (decision element) to ensure data conversion without disturbing the pre-programmed polarization-state of the Fe-FinFETs in the proposed ADC. Our extensive analysis utilizing an experimentally calibrated compact model for Fe-FinFETs and the 7-nm ASAP PDK from ARM for CMOS neuron indicates that the proposed feedforward LTNN ADC consumes $5.44 \mu \mathrm{W}$ of power and $1.03 \mu \mathrm{m}^{2}$ of area while operating at a speed of 1.23 megasamples per second for a 4-bit precision which is sufficient for practical neuromorphic inference accelerators [24].

The manuscript is organized as follows: the architecture of the feedforward LTNN ADC is discussed in section II. The structure and modeling approach for Fe-FinFETs are described in section III. The results of our extensive analysis of the proposed feedforward neural network ADC exploiting Fe-FinFETs for performance metrics such as area, speed and power consumption are presented in section IV and the conclusions are drawn in section V.

\section{FEedForward LTNN ADC ARChitecture}

The schematic view of a feedforward LTNN ADC is shown in Fig. 1. It consists of a grid of resistive elements $T_{S 0, \ldots, 3}$ and $T_{R 0, \ldots, 3}$, synapses (weights) $T_{10, \ldots, 32}(n(n+1) / 2$ for $n$ - bit ADC) and neurons ( $n$ decision elements for $n$-bit ADC). Each neuron is fed the analog voltage to be converted, $V_{S}$, via $T_{S 0, \ldots, 3}$, a negative reference voltage $V_{R}$ via $T_{R 0, \ldots, 3}$, and the (complementary) outputs from other neurons $V_{1}, V_{2}, V_{3}, V_{4}$ through the synaptic elements $T_{10, \ldots, 32}$. The input voltage $V_{S}$ is converted to a digital output with $V_{0}$ as the LSB and $V_{3}$ as the MSB. The feedforward LTNN ADC implementation works on the principle of successive approximation [11], where the MSB is first determined and the information is then used to determine the lower significant bits. Since a lower significant bit does not influence a more significant bit, the synaptic elements are not used in the lower triangular portion of the grid. This results in a feedforward neural network which eliminates the issues with the Hopfield ADCs with feedback such as oscillations and metastability of the output.

The dynamics of the LTNN ADC can be described by

$$
C \dot{U}_{j}=-\sum_{i=j+1}^{N-1} T_{i j} V_{i}-T_{j} U_{j}+I_{j}
$$

where

$$
\begin{aligned}
I_{j} & =T_{S j} V_{S}-T_{R j} V_{R} \\
T_{j} & =T_{S j}+T_{R j}+\sum_{i=j+1}^{N-1} T_{i j} \\
V_{j} & =g\left(U_{j}\right)
\end{aligned}
$$

where $C$ is the input capacitance of the neuron circuit, $N$ is the number of bits of the ADC and $g(\cdot)$ represents the transfer function of the neuron. The optimal set of conductance values for the resistive elements and the synapses can be obtained as [12]:

$$
T_{i j}=2^{i}, \quad T_{R i}=2^{i}, \quad T_{S i}=1 .
$$

It may be noted that the implementation of synapses is critical to the area and power consumption of the proposed ADC since the number of synapses scales as $O\left(n^{2}\right)$ for an $n$-bit ADC, compared to the number of neurons $(O(n))$. Therefore, we propose to implement the synapses (and resistive elements) using Fe-FinFETs with ultra-low area and high scalability.

Also, the update rule for the $i$-th neuron can be given as:

$$
\begin{aligned}
V_{i} & =1 \quad \text { if }\left(V_{S}-\sum_{j=i+1}^{3} 2^{j} V_{j}\right) \geq 2^{i} \\
& =0 \quad \text { otherwise. }
\end{aligned}
$$

The neurons act as decision elements and generate a feedback voltage $\bar{V}_{i}$ which is applied to the synapses. We have designed a differential amplifier based neuron as shown in Fig. 2 to ensure data conversion without altering the pre-programmed polarization-state of the Fe-FinFETs in the proposed ADC. The final digital output is generated from $\bar{V}_{i}$ using a CMOS inverter.

\section{Fe-FinfET Structure and Modeling Approach}

The 3D view of the ferroelectric FinFET is shown in Fig. 3(a). It consists of an additional ferroelectric layer of dopedhafnium oxide $\left(\mathrm{HfZrO}_{\mathrm{x}}\right)$ in the gate stack. The polarizationstate of the ferroelectric layer dictates the threshold voltage 


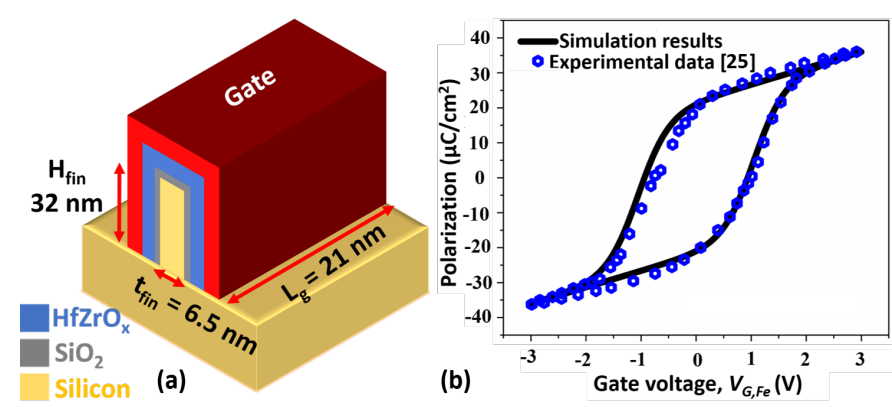

Fig. 3. (a) 3D-view of the Fe-FinFET with $\mathrm{HfZrO}_{x}$ ferroelectric layer and (b) Calibration of compact model parameters by reproducing the experimental results of $\mathrm{HfZrO}_{\mathrm{x}}$ ferroelectric capacitor [25]

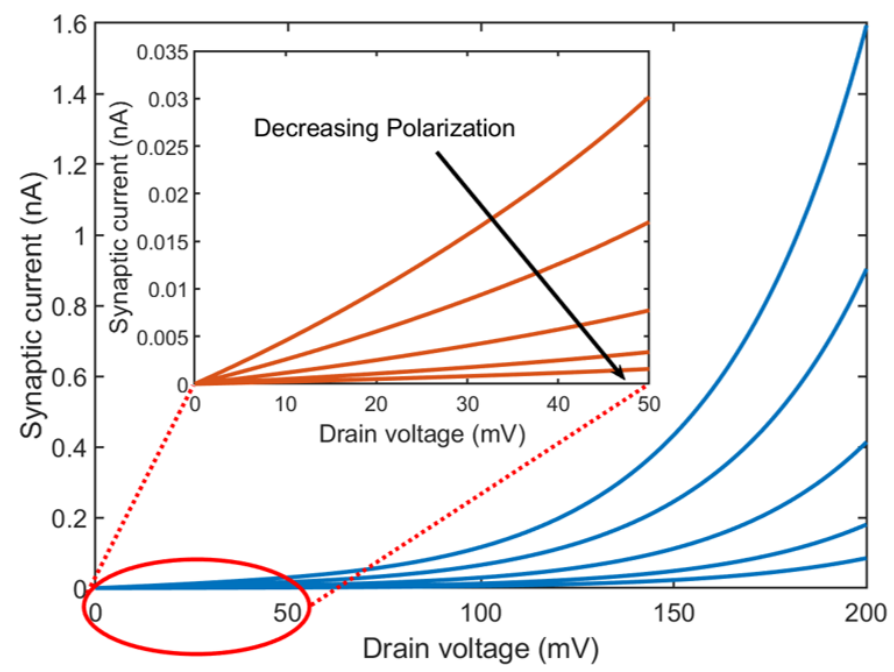

Fig. 4. Output characteristics of the Fe-FinFET synapse for different polarization-states.

and may be tuned with the help of electrical pulses applied at the gate and drain terminals [23], [26], [27]. $\mathrm{HfZrO}_{\mathrm{x}}$-based ferroelectric FETs with a capability of tuning the weights (conductance states) with more than 5-bit precision have already been experimentally demonstrated [25], [27]. In this work, we have utilized an experimentally calibrated compact model for Fe-FinFETs [28] which accurately captures the static polarization characteristics, program/erase behavior, temporal dynamics during transient analysis and the history effect of a $\mathrm{HfZrO}_{\mathrm{x}}$ stack [26]. The parameters of the compact model have been tuned to reproduce the experimental characteristics of the $\mathrm{HfZrO}_{\mathrm{x}}$-based ferroelectric capacitor reported in [25] and shown in Fig. 3(b).

The output characteristics of the proposed Fe-FinFET synapse (where Fe-FinFET is in the diode-connected configuration as shown in Fig. 1) for different polarization-states of the ferroelectric $\mathrm{HfZrO}_{\mathrm{x}}$ layer, obtained using the experimentally calibrated compact model [28], are shown in Fig. 4. As can be observed from Fig. 4, the output characteristics of the proposed Fe-FinFET synapse are highly linear for lower range of drain voltages $\left(V_{D}<50 \mathrm{mV}\right)$ which facilitates the application of Fe-FinFETs as efficient synapses and resistive elements in the proposed LTNN ADC.
TABLE I

NONLINEARITY OF ADC OUTPUT

\begin{tabular}{|c|c|c|}
\hline No. of bits & Max differential nonlinearity & Max integral nonlinearity \\
\hline 2 & $0.008 \mathrm{LSB}$ & $0.005 \mathrm{LSB}$ \\
3 & $0.18 \mathrm{LSB}$ & $0.14 \mathrm{LSB}$ \\
4 & $0.28 \mathrm{LSB}$ & $0.24 \mathrm{LSB}$ \\
5 & $0.62 \mathrm{LSB}$ & $0.45 \mathrm{LSB}$ \\
6 & $1.18 \mathrm{LSB}$ & $1.09 \mathrm{LSB}$ \\
\hline
\end{tabular}

TABLE II

PERformanCE Metrics

\begin{tabular}{|c|c|c|c|}
\hline No. of bits & Area $\left(\mu \mathrm{m}^{2}\right)$ & Speed $($ samples/s) & Energy/sample $(\mathrm{pJ})$ \\
\hline 2 & 0.31 & $7.09 \times 10^{5}$ & 3.84 \\
3 & 0.52 & $1.12 \times 10^{6}$ & 3.63 \\
4 & 0.76 & $1.23 \times 10^{6}$ & 4.42 \\
5 & 1.03 & $7.94 \times 10^{5}$ & 8.57 \\
6 & 1.34 & $4.67 \times 10^{6}$ & 1.75 \\
\hline
\end{tabular}

\section{RESUlTS AND Discussion}

Utilizing the experimentally calibrated compact model for $\mathrm{Fe}-$ FinFETs [28] and the 7-nm technology PDK from ARM [29] for CMOS circuits, we have performed an extensive analysis of the proposed LTNN ADC (Fig. 1) based on the FeFinFET synapses and the neuron circuitry shown in Fig. 2. The polarization-state of the Fe-FinFET synapses (and resistive elements) are first tuned to program their conductance value to the desired values obtained from equation (5). The neuron circuit is designed such that the maximum voltage drop across the Fe-FinFETs is $50 \mathrm{mV}$. This ensures proper operation of the proposed ADC without changing the pre-programmed polarization-state and conductance of the Fe-FinFET synapses and resistive elements during data conversion. In addition, a low voltage across the Fe-FinFET synapses also leads to higher linearity in their output characteristics leading to an efficient ADC design. Low-to-medium precision LTNN ADCs based on Fe-FinFET synapses were designed following this approach.

The simulation results for the output of the proposed $\mathrm{Fe}$ FinFET based LTNN ADC with different output-bit precision are shown in Fig. 5. As can be observed from Fig. 5, the outputs obtained via simulations deviate from the ideal ADC characteristics as the output-bit precision increases.

Furthermore, to quantify the accuracy of the proposed LTNN ADC, we have used two standard metrics: differential and integral non-linearity. While differential non-linearity (DNL) is the difference between the step width of the ADC output and the ideal ADC characteristics, the integral nonlinearity (INL) is the deviation of the ADC transfer function from the transfer function of an ideal ADC i.e. a straight line. The maximum values of the differential and integral nonlinearity for the proposed ADC are outlined in Table I. Both DNL and INL increase and the accuracy degrades for the proposed Fe-FinFET based LTNN ADC with an increase in the output-bit precision.

Moreover, we have also analysed important performance metrics such as area, speed and power consumption of the proposed LTNN ADC for different output-bit precisions (Table II) and compared them against state-of-the-art ADCs from 

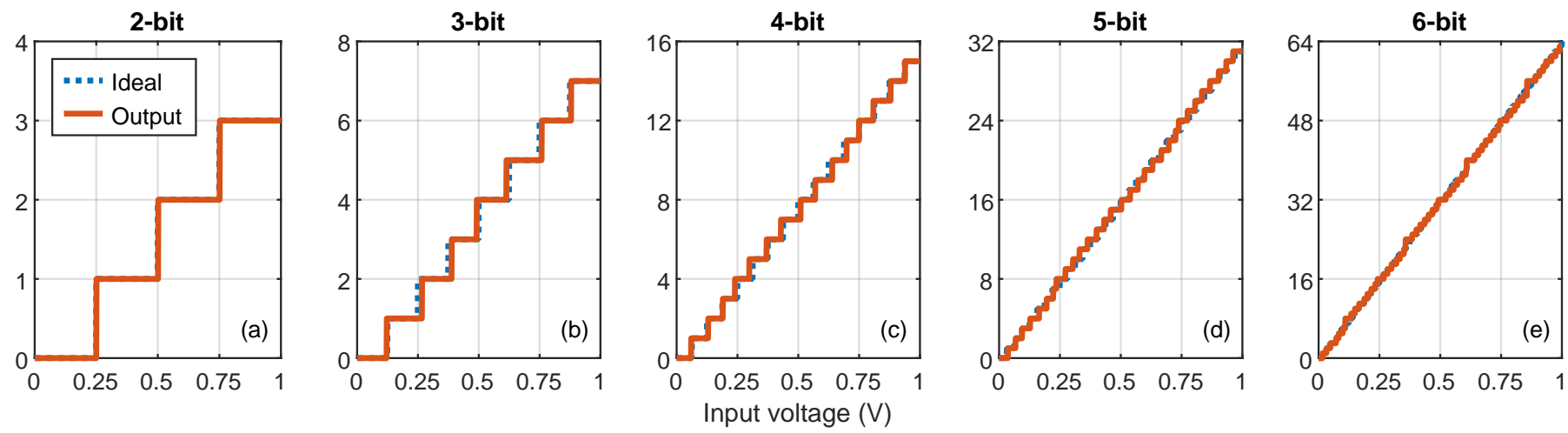

Fig. 5. The simulated ADC output for different output-bit precision: (a) 2-bit, (b) 3-bit, (c) 4-bit, (d) 5-bit, (e) 6-bit.

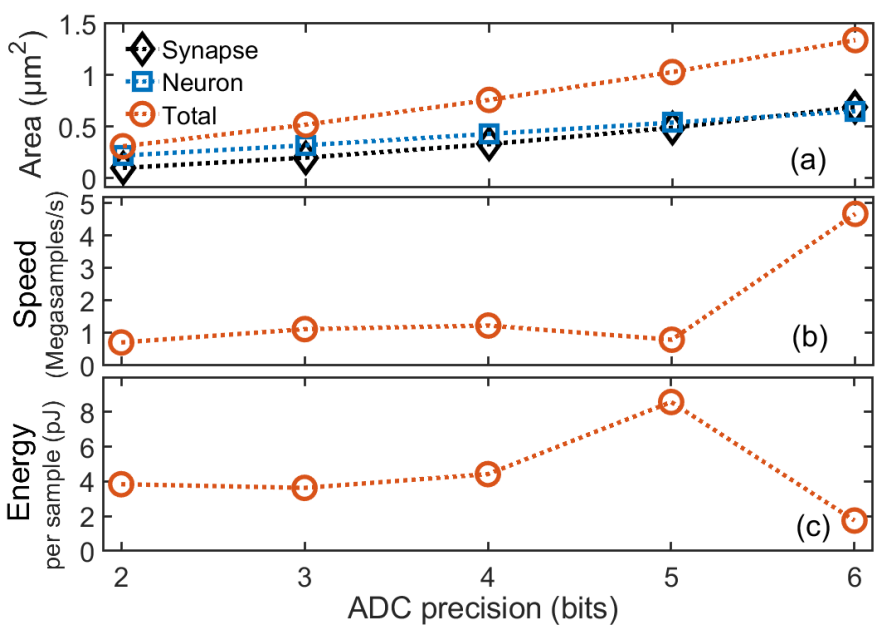

Fig. 6. Performance metrics of the proposed ADC: (a) area, (b) speed and (c) energy.

a comprehensive survey [30].

\section{A. Area}

The area of the proposed ADC with different output-bit precision was obtained from the layouts using the 7-nm technology PDK from ARM. The baseline FinFET from the PDK was utilized for representing Fe-FinFETs in the layout. The total area of the proposed ADC for different output-bit precision are reported in Table II. Furthermore, the contribution of the neurons and synaptic elements in the areal landscape is also shown in Table III. The area occupied by the synapses increases quadratically with increasing output-bit precision, while the area occupied by the neurons increases linearly. Also, the synapses dominate the areal landscape for the proposed ADC with higher output-bit precision ( $>$ 5-bit) as shown in Fig. 6(a). Therefore, utilization of highly scalable and ultra-compact Fe-FinFETs as synaptic elements provides an inherent scaling-benefit. Moreover, the silicon footprint for the proposed Fe-FinFET based LTNN ADC with a 6-bit precision is $1.34 \mu \mathrm{m}^{2}$, which is significantly smaller as compared to the conventional ADC [31] with lowest area $\left(580 \mu \mathrm{m}^{2}\right)$ reported in the comprehensive survey of ADCs [30].
TABLE III

AREA OCCUPIED BY NEURONS AND SYNAPSES

\begin{tabular}{|c|c|c|}
\hline No. of bits & Neuron area $\left(\mu \mathrm{m}^{2}\right)$ & Synapse area $\left(\mu \mathrm{m}^{2}\right)$ \\
\hline 2 & 0.22 & 0.10 \\
3 & 0.32 & 0.20 \\
4 & 0.43 & 0.33 \\
5 & 0.54 & 0.49 \\
6 & 0.65 & 0.69 \\
\hline
\end{tabular}

TABLE IV

POWER CONSUMED BY NEURONS AND SYNAPSES

\begin{tabular}{|c|c|c|}
\hline No. of bits & Neuron power $(\mu \mathrm{W})$ & Synapse power $(\mathrm{pW})$ \\
\hline 2 & 2.72 & 0.76 \\
3 & 4.08 & 3.37 \\
4 & 5.44 & 8.99 \\
5 & 6.80 & 11.9 \\
6 & 8.16 & 153 \\
\hline
\end{tabular}

\section{B. Speed}

We also investigated the speed (in terms of the number of input voltage samples that can be processed per unit time) of the proposed ADC with different output bit precision as shown in Fig. 6(b). We observe that the speed of the proposed ADC increases significantly as the output-bit precision increases from 5-bit to 6-bit. This is attributed to the technique used for mapping the required Fe-FinFET synaptic resisances for the LTNN ADC. According to equation (5), the resistances corresponding to the synapses in the LTNN ADC should be tuned in the ratio $1: 2: \ldots: 2^{n-1}$. While tuning the polarization-states of the Fe-FinFETs to realize the synaptic resistances that fit best to this particular ratio for 6-bit ADC, we found that majority of the Fe-FinFET synapses exhibit a lower resistance value. This results in a lower time constant $(R C)$ and a sharp increase in the speed for 6-bit ADC. Whereas for the 5-bit ADC design, after tuning the polarization-states for Fe-FinFETs to fit the required resistance ratio, we found that most of the synapses exhibit higher resistances leading to a reduced speed.

\section{Power Consumption}

The power consumed by the neurons and synapses of the proposed Fe-FinFET based LTNN ADC with different output- 
bit precision are shown in Table IV. Although the power consumed by the synaptic elements increases quadratically with increasing output-bit precision of the ADC, it is considerably small as compared to the power consumed by the neuron circuitry (which increases linearly with output-bit precision). The extremely high energy-efficiency of the FeFinFET synapses is attributed to the low voltage drop across them during the conversion operation. Moreover, similar to the ADC speed, there is a steep increase in the power consumption of the synapses (11.9 pW to $153 \mathrm{pW})$ as the output-bit precision increases from 5-bit to 6-bit. As explained in section IV-B, majority of Fe-FinFET synapses are tuned to the low resistance-state for output-bit precision of 6-bits leading to a higher power consumption.

The energy consumed (per input sample) by the proposed ADC exhibits a non-monotonic behavior with the output-bit precision as shown in Fig. 6(c). While the power consumed by the neuron circuit which dominates the energy landscape of the proposed ADC increases linearly with the outputbit precision, the speed of the ADC also increases leading to a reduced conversion time per sample with increasing output-bit precision. The simultaneous increase in the power consumption and reduction in the conversion time results in a non-monotonic behavior of the energy consumed by the proposed ADC. Compared to the state-of-the-art ADCs from the survey [30], the proposed 6-bit Fe-FinFET based LTNN ADC has a similar energy consumption per sample as [32], but outperforms it in terms of both area and speed: $2 \mathrm{~mm}^{2}$ and $100 \mathrm{kilosamples} / \mathrm{s}$ for [32] versus $1.34 \mu^{2}$ and 4.67 megasamples/s for the proposed 6-bit ADC.

\section{CONCLUSION}

In this work, we have designed an ultra-compact and highly scalable low-to-medium precision neural network based ADC with Fe-FinFETs as synaptic elements. Our extensive analysis indicates that the proposed ADC consumes $5.44 \mu \mathrm{W}$ of power and $1.03 \mu^{2}$ of area while operating at a speed of 1.23 megasamples per second for a 4-bit precision and could be a lucrative alternative to the conventional ADCs specifically for neuromorphic processing engines and neuro-optimization circuits based on cross-point array of emerging non-volatile memories.

\section{REFERENCES}

[1] H. Tsai et al., "Recent progress in analog memory-based accelerators for deep learning," Journal of Physics D: Applied Physics, vol. 51, no. 28, p. 283001, jun 2018.

[2] T. P. Xiao et al., "Analog architectures for neural network acceleration based on non-volatile memory," Applied Physics Reviews, vol. 7, no. 3, p. 031301, 2020.

[3] H. Jiang et al., "Analog-to-digital converter design exploration for compute-in-memory accelerators," IEEE Design \& Test, pp. 1-1, 2021.

[4] M. Hu et al., "Dot-product engine for neuromorphic computing: Programming $1 \mathrm{~T} 1 \mathrm{M}$ crossbar to accelerate matrix-vector multiplication," in 2016 53nd ACM/IEEE Design Automation Conference, 2016, pp. 1-6.

[5] M. J. Marinella et al., "Multiscale co-design analysis of energy, latency, area, and accuracy of a ReRAM analog neural training accelerator," IEEE J. Emerg. and Sel. Topics in Circuits and Systems, vol. 8, no. 1, pp. 86-101, 2018

[6] S. Sahay et al., "Energy-efficient moderate precision time-domain mixed-signal vector-by-matrix multiplier exploiting 1T-1R arrays," IEEE J. Exploratory Solid-State Computational Devices and Circuits, vol. 6 , no. 1 , pp. $18-26,2020$
[7] M. Bavandpour et al., "3d-aCortex: an ultra-compact energy-efficient neurocomputing platform based on commercial 3d-NAND flash memories," Neuromorphic Computing and Engineering, vol. 1, no. 1, p. 014001, jul 2021.

[8] S. Sahay et al., "A 2T-1R cell array with high dynamic range for mismatch-robust and efficient neurocomputing," in 2020 IEEE Int. Memory Workshop, 2020, pp. 1-4.

[9] M. Bavandpour et al., "Mixed-signal vector-by-matrix multiplier circuits based on 3d-nand memories for neurocomputing," in 2020 Design, Automation Test in Europe Conference Exhibition, 2020, pp. 696-701.

[10] D. Tank and J. Hopfield, "Simple 'neural' optimization networks: An A/D converter, signal decision circuit, and a linear programming circuit," IEEE Trans. Circuits and Systems, vol. 33, no. 5, pp. 533-541, 1986.

[11] V. Chande and P. Poonacha, "On neural networks for analog to digital conversion," IEEE Trans. Neural Networks, vol. 6, no. 5, pp. 1269-1274, 1995.

[12] X. Guo et al., "Modeling and experimental demonstration of a hopfield network analog-to-digital converter with hybrid CMOS/memristor circuits," Frontiers in Neuroscience, vol. 9, p. 488, 2015.

[13] G. Indiveri et al., "Neuromorphic silicon neuron circuits," Frontiers in Neuroscience, vol. 5, 2011.

[14] L. Danial et al., "A pipelined memristive neural network analog-todigital converter," in 2020 IEEE Int. Symposium on Circuits and Systems, 2020, pp. 1-5.

[15] S. Hu et al., "Associative memory realized by a reconfigurable memristive Hopfield neural network," Nat Commun, vol. 6, p. 7522, 2015.

[16] M. R. Mahmoodi et al., "Versatile stochastic dot product circuits based on nonvolatile memories for high performance neurocomputing and neurooptimization," Nat Commun, vol. 10, p. 5113, 2019.

[17] L. Danial et al., "Logarithmic neural network data converters using memristors for biomedical applications," in 2019 IEEE Biomedical Circuits and Systems Conference, 2019, pp. 1-4.

[18] S. B. Eryilmaz et al., "Brain-like associative learning using a nanoscale non-volatile phase change synaptic device array," Frontiers in Neuroscience, vol. 8, 2014.

[19] S. Sahay and M. Suri, "Recent trends in hardware security exploiting hybrid CMOS-resistive memory circuits," Semiconductor Science and Technology, vol. 32, no. 12, p. 123001, oct 2017.

[20] T. S. Böscke et al., "Ferroelectricity in hafnium oxide: CMOS compatible ferroelectric field effect transistors," in 2011 Int. Electron Devices Meeting, 2011, pp. 24.5.1-24.5.4.

[21] J. Müller et al., "Ferroelectricity in $\mathrm{HfO} 2$ enables nonvolatile data storage in 28 nm HKMG," in 2012 Symp. on VLSI Technology, 2012, pp. $25-26$

[22] K. Chatterjee et al., "Self-aligned, gate last, FDSOI, ferroelectric gate memory device with 5.5 -nm $\mathrm{Hf} 0.8 \mathrm{Zr} 0.2 \mathrm{O} 2$, high endurance and breakdown recovery," IEEE Electron Device Letters, vol. 38, no. 10, pp. 13791382, 2017.

[23] P. Wang et al., "Drain-erase scheme in ferroelectric field-effect transistor-part I: Device characterization," IEEE Trans. Electron Devices, vol. 67, no. 3, pp. 955-961, 2020.

[24] J. Choi et al., "PACT: parameterized clipping activation for quantized neural networks," 2018.

[25] S. Oh et al., "HfZrOx-based ferroelectric synapse device with 32 levels of conductance states for neuromorphic applications," IEEE Electron Device Letters, vol. 38, no. 6, pp. 732-735, 2017.

[26] P. Wang et al., "Investigating ferroelectric minor loop dynamics and history effect-part i: Device characterization," IEEE Trans. Electron Devices, vol. 67, no. 9, pp. 3592-3597, 2020.

[27] M. Jerry et al., "Ferroelectric FET analog synapse for acceleration of deep neural network training," in 2017 IEEE Int. Electron Devices Meeting, 2017, pp. 6.2.1-6.2.4.

[28] A. Gaidhane, R. Dangi, S. Sahay, A. Verma, and Y. Chauhan, "A computationally efficient compact model for ferroelectric FinFETs switching with asymmetric non-periodic input signals," Jan 2022. [Online]. Available: TechRxiv:10.36227/techrxiv.18095684.v1

[29] L. T. Clark et al., "ASAP7: A 7-nm finFET predictive process design kit," Microelectronics Journal, vol. 53, pp. 105-115, 2016.

[30] B. Murmann, "ADC performance survey 1997-2021." [Online]. Available: http://web.stanford.edu/ murmann/adcsurvey.html

[31] K. D. Choo et al., "Area-efficient 1GS/s 6b SAR ADC with chargeinjection-cell-based DAC," in 2016 IEEE Int. Solid-State Circuits Conference, 2016, pp. 460-461.

[32] D. C. Daly and A. P. Chandrakasan, "A 6b 0.2-to-0.9V highly digital flash ADC with comparator redundancy," in 2008 IEEE Int. Solid-State Circuits Conference, 2008, pp. 554-635. 